\title{
Role of the RUNX2 p.R225Q mutation in cleidocranial dysplasia: a rare presentation and an analysis of the RUNX2 protein structure
}

\author{
L.Z. Wu ${ }^{1}$, W.Q. Su${ }^{2}$, Y.F. Liu' ${ }^{1}$ X. Ge ${ }^{1}$, Y. Zhang ${ }^{1}$ and X.J. Wang ${ }^{1}$ \\ ${ }^{1}$ Department of Pediatric Dentistry, School of Stomatology, \\ The Fourth Military Medical University, Xi'an, China \\ ${ }^{2}$ Third Military Medical University, Chongqing, China \\ Corresponding author: X.J. Wang \\ E-mail: wxjingpd@hotmail.com
}

Genet. Mol. Res. 13 (1): 1187-1194 (2014)

Received December 18, 2012

Accepted July 23, 2013

Published February 27, 2014

DOI http://dx.doi.org/10.4238/2014.February.27.3

\begin{abstract}
Cleidocranial dysplasia (CCD; MIM 119600) is an autosomal dominant hereditary disorder of skeletal features whose characteristic clinical symptoms are caused by mutations in the RUNX2 gene. Varying degrees of clavicular hypoplasia and dental abnormalities are the most prominent features of this disorder. In this study, we presented a Chinese family that included 4 individuals with a p.R225Q mutation in the RUNX2 gene and characteristic CCD phenotypes. Through structural analysis of the p.R225Q mutation in the RUNX2 protein, we determined that the location of this mutation has the potential to affect DNA binding by RUNX2. The proband in this CCD-affected family showed a specific clinical phenotype of CCD that included a median pseudo-cleft palate, which is a presentation of this mutation that has not been reported previously. On the basis of the structural analysis, this study further demonstrated that the p.R225Q mutation abolished DNA binding by RUNX2 and its results also suggested that other genetic and/ or environmental factors could affect the CCD phenotypes.
\end{abstract}

Key words: Cleidocranial dysplasia; Median pseudo-cleft palate; RUNX2; R225 


\section{INTRODUCTION}

Cleidocranial dysplasia (CCD) is an autosomal dominant disorder characterized by skeletal and dental abnormalities. It has a prevalence of $<1$ per 1 million people. In 1997, RUNX2 was identified as the CCD-causing gene (Lee et al., 1997; Mundlos et al., 1997; Otto et al., 1997). The main clinical features of CCD include hypoplastic or absent clavicles, delayed closure of cranial fontanels and sutures, wormian bones, frontal bossing, supernumerary and delayed-erupting teeth, and wide pubic symphysis (Mundlos, 1999). Some degree of clavicular hypoplasia and dental abnormalities are the most consistent features of this disease (Quack et al., 1999), whereas median pseudo-cleft palate in CCD patients for the p.R225Q mutation in RUNX2 has not been reported previously in the literature. Moreover, although functional studies have suggested that mutations at this codon interfere with nuclear localization and abolish DNA binding (Quack et al., 1999; Yoshida et al., 2002), the R225 structure in RUNX2 has never been analyzed before.

In this study, we investigated CCD in a Chinese family. Direct DNA sequencing of the entire coding region and the exon-intron boundaries in RUNX2 identified the missense mutation p.R225Q in the Runt domain of the RUNX2 protein. We identified a rare presentation of this mutation and analyzed the specific structure of R225 in RUNX2.

\section{PATIENTS AND METHODS}

This study was approved without restrictions by the Research Ethics Committee of School of Stomatology, Fourth Military Medical University, and authorized by all individuals and patients.

\section{Patients}

Four affected individuals of a Chinese family with the characteristic clinical diagnosis of $\mathrm{CCD}$ were included in this study. Their clinical presentation is described below and the pedigree is illustrated in Figure 1. The proband (IV1) was an 8-year-old boy, who is indicated with an arrow.

\section{Genetic testing}

Blood ( $3 \mathrm{~mL}$ ) was drawn from all CCD-affected individuals, the healthy father of the proband, and 100 unrelated normal controls. Genomic DNA was extracted from whole blood using the TIANamp Blood DNA Kit [Tiangen Biotech, Co. Ltd., Beijing, China]. Exons $0-7$ of the RUNX2 gene were amplified by PCR with the primers given in Table 1. PCR amplifications were performed in a thermal cycler (PE 2400, PE Biosystems) in $50 \mu \mathrm{L}$ reaction volumes; $100 \mathrm{ng}$ genomic DNA and $2.0 \mu \mathrm{L}$ of each primer $(10 \mu \mathrm{M})$ were mixed with $25 \mu \mathrm{L} 2 \mathrm{X}$ PCR mastermix. Automated sequencing was performed on an ABI 377 automatic sequencer. DNA sequences were analyzed using the BLASTN program at NCBI (http://www. ncbi.nlm.nih.gov/BLAST). The mutation was confirmed by sequencing of the products from several independent PCRs. After a mutation within an exon 3 was detected in the affected individuals, this exon was specifically PCR amplified in the father of the proband and 100 unrelated normal controls. 
Table 1. Primers used for polymerase chain reaction, sequencing reactions, and amplification conditions.

\begin{tabular}{lllcc}
\hline Exon & Sense $\left(5^{\prime}-3^{\prime}\right)$ & Antisense $\left(5^{\prime}-3^{\prime}\right)$ & Fragment size $(\mathrm{bp})$ & Annealing $\left({ }^{\circ} \mathrm{C}\right)$ \\
\hline 0 & TACCCAGCCACCGAGACCAACAGAG & GTTTTGCTGACATGGTGTCAC & 341 & 60 \\
1 & ACTTGTGGCTGTTGTGATGC & GCCGCCAAGGCAGGA & 513 & 57 \\
2 & CAGATGCTTGATTCCTGTCGG & GTGCTGATTTGTATACAGACTAG & 391 & 56 \\
3 & TCATTGCCTCCTTAGAGATGC & GGACATGAAAGTGACACTAAC & 310 & 60 \\
4 & TATAAAGCAATTTGAAATGCAAGG & GTTTTGAAGTGAACACATCTCC & 429 & 54 \\
5 & TAAGGCTGCAATGGTTGCTAT & GTCACTGTGAGCATGGATGAG & 306 & 59 \\
6 & TAGAACATTAGAGCTGGAAGG & CGGACAGTAACAACCAGACAG & 188 & 60 \\
7 & TGTGGCTTGCTGTTCTTTATG & GATACCACTGGGCCACTGCT & 630 & 60 \\
\hline
\end{tabular}

\section{RESULTS}

\section{Clinical findings}

A Chinese family including 4 affected individuals with the clinical diagnosis of CCD was investigated. The genetic pedigree is shown in Figure 1. The proband (IV1) was an 8-yearold boy, who is indicated by the arrow. IV1 showed the characteristic features of CCD, including a concave face type, an open anterior fontanel (the anterior fontanel normally closes at 12-18 months of age), bilaterally absent clavicles, a sloping shoulder, and maxillary hypoplasia (Figure 2A-D). He had primary dentition with the lower left first molar erupted; the palate was highly arched with a median pseudo-cleft (Figure 2E). His height $(120 \mathrm{~cm})$ was in the 5 th percentile for his age group (the normal range for 8-year-old boys is 121.6-132.2 cm), whereas his weight $(22.5$ $\mathrm{kg}$ ) was within the normal range (22.2-30.0 kg). His panoramic view showed one supernumerary tooth in the mandible and another in the maxillary area; the primary teeth overlapped with the secondary teeth instead of showing degeneration (Figure 2F). His mother, aged 35 years, was also of short stature $(148 \mathrm{~cm})$, had a body weight of $50 \mathrm{~kg}$, and her panoramic view showed one supernumerary tooth in the left mandible and the other in the right mandible (Figure 2G). His uncle, aged 33 years, was of short stature $(152 \mathrm{~cm})$, had a body weight of $56 \mathrm{~kg}$, and his panoramic view showed one supernumerary tooth in the left mandible (Figure $2 \mathrm{H})$. His grandmother, aged 58 years, was of short stature $(147 \mathrm{~cm})$, had a body weight of $42 \mathrm{~kg}$, and her panoramic view showed one supernumerary tooth in the left mandible (Figure 2I). Chest radiographs of these 3 relatives of the proband showed bilaterally absent clavicles (Figure 3A-C), and their facial features resembled those of the proband. The proband's father was healthy without any phenotypical signs of CCD.

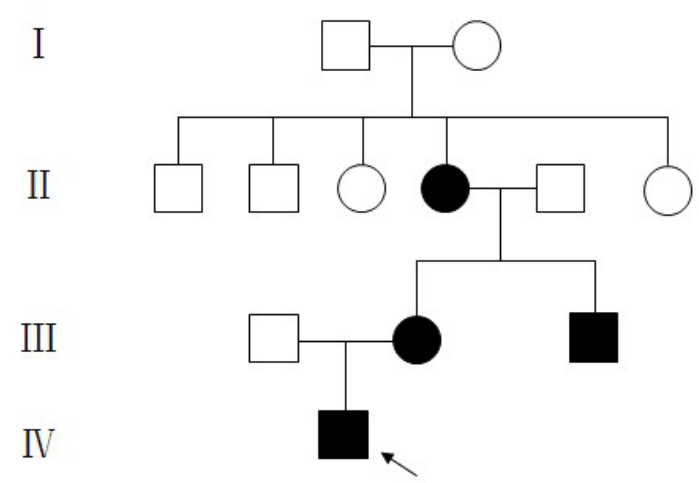

Figure 1. Genetic pedigree of a Chinese family with cleidocranial dysplasia (proband is indicated with an arrow). 


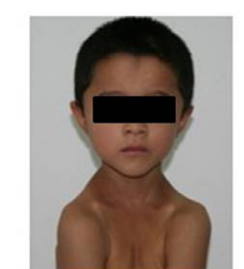

A

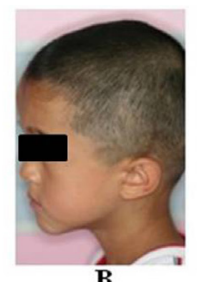

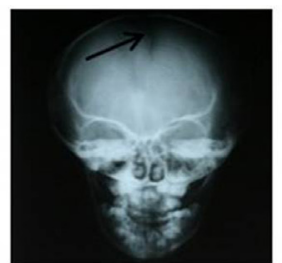

C

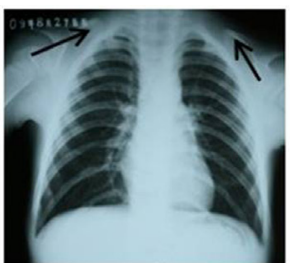

D

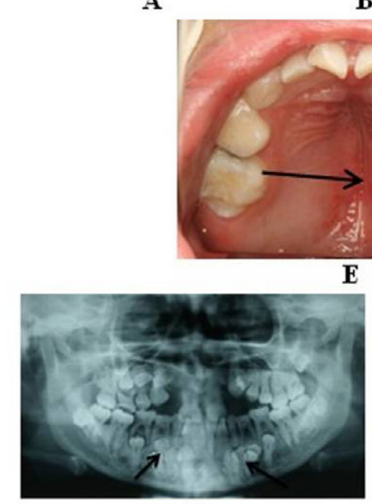

G

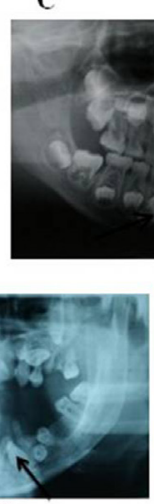

$\mathbf{H}$

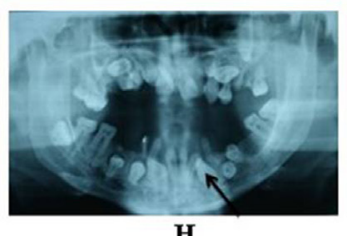

ave face

fype, narrow and sloping shoulders. B. Photograph of the proband shows hypoplasia of the maxilla, frontal bossing. C. Cranial X-ray showing open anterior fontanel, and wormian bones near the lambdoid suture. D. Chest radiograph showing small and bell-shaped thorax with short and oblique ribs, and bilaterally absent clavicles. E. Oral manifestations of the proband to show that the palate was highly arched with a pseudo-cleft. F.-I. Panoramic view showing retention of deciduous teeth, impacted permanent teeth and supernumerary teeth. G. Indicates proband. H. Indicates his mother. I. Indicates his grandmother.

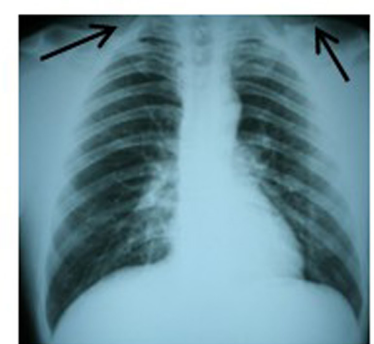

A

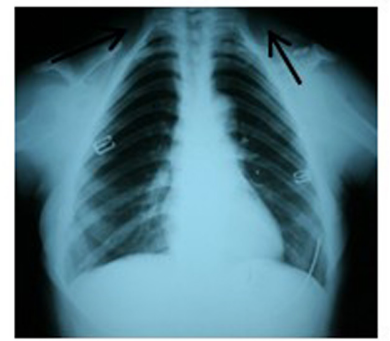

C

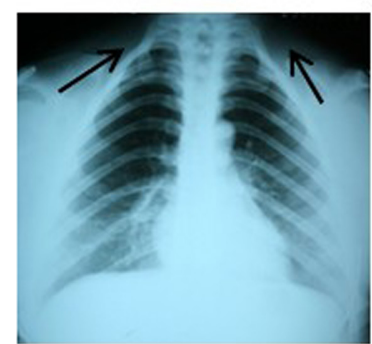

B

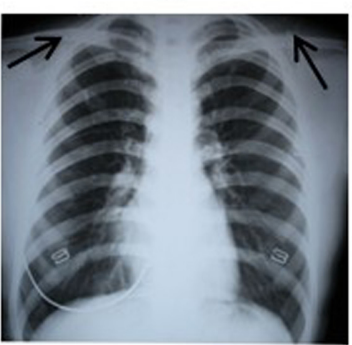

D

Figure 3. Chest radiograph shows bilaterally absent clavicles of the proband (A), his mother $(\mathbf{B})$, his grandmother $(\mathbf{C})$, and his father (D). 


\section{Genetic analysis}

Genomic DNA was extracted from whole blood from the proband and his mother, grandmother, and healthy father, as well as from 100 unrelated normal controls. The RUNX2 gene was studied by PCR and direct sequencing of the entire coding region and the exon-intron boundaries of the gene. A missense mutation (c.674G>A, R225Q) (Figure 4), was identified in exon 3, which was predicted to substitute an arginine with a glutamine residue at codon 225 of the RUNX2 protein. This mutation was found in all of the CCD-affected individuals, but was not present in the proband's healthy father or in the 100 unrelated normal controls.
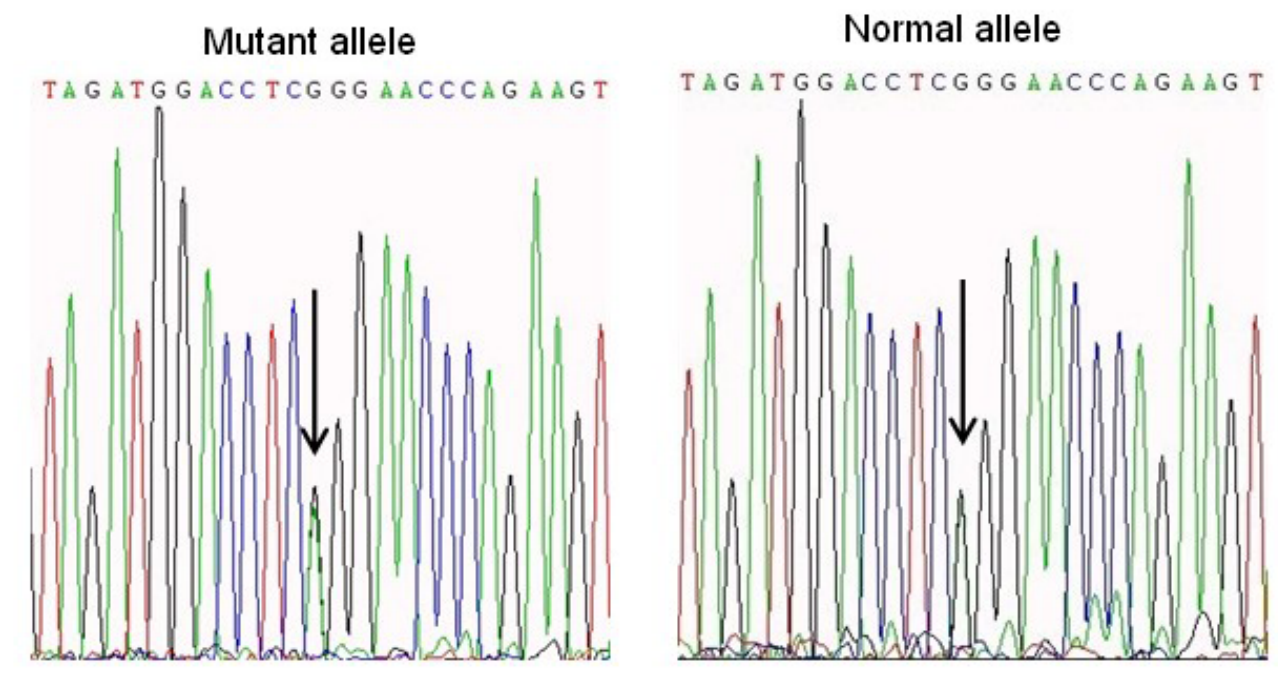

Figure 4. Sequencing results show the heterozygous mutant allele with $\mathrm{G}>\mathrm{A}$ missense mutation in c.674G.

The arginine at the codon 225 position of RUNX2 is conserved in the Runt-domain proteins of all vertebrates, suggesting that this arginine residue is functionally important (Figure 5A). The wild-type and mutated 3D models of the RUNX2 Runt domain were built with the geno3D server by using $1 \mathrm{HJC}$ as a template (Combet et al., 2002). Structures were displayed and analyzed in the pdbviewer software. The wild-type and mutated models are shown in blue and pink colors, respectively. The location of R225 is marked in yellow and that of Q225 in green. The DNA fragment is shown in red. The structures indicated that the p.R225Q mutation is located closely to the region of Runt for binding of RUNX2 to the DNA helix (Figure 5B).

\section{DISCUSSION}

In this study, all affected individuals were diagnosed with $\mathrm{CCD}$ on the basis of clinical findings and genetic analysis. The affected individuals showed features characteristic of CCD, such as absent clavicles, open anterior fontanel, short stature, delayed eruption of permanent teeth, and supernumerary teeth. They also carried the missense mutation (c.674G $>$ A, R225Q) in exon 3 of the RUNX2 gene. The proband's healthy father and 100 unrelated healthy con- 


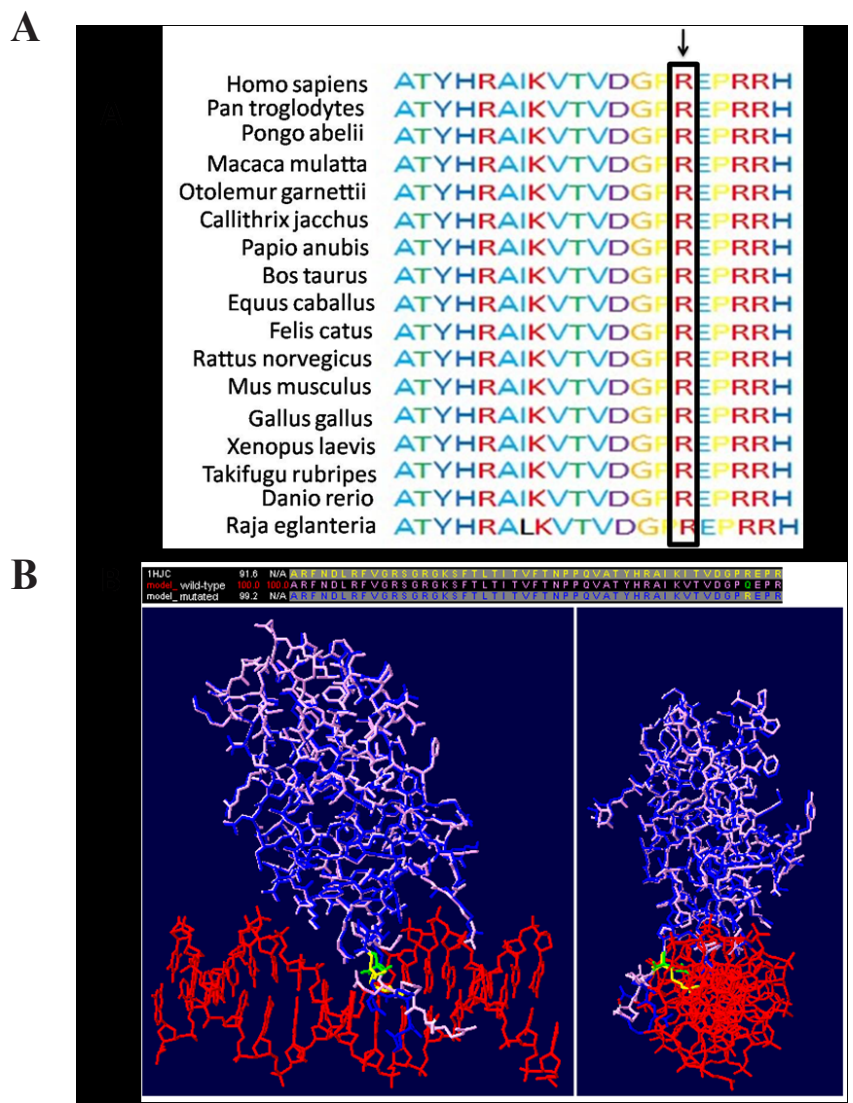

Figure 5. A. Comparison of sequences in the vicinity of RUNX2 R225 (arrow) from various species. B. The wild-type and mutated models are shown in blue and pink color, respectively. The location of R225 is yellow and Q225 is green. DNA fragment is red. Structrures show that the mutation p.R225Q locates closely to the region of Runt for binding to DNA helix.

trols did not carry this mutation, suggesting that it is of significance for the observed pathologies. This mutation has been reported in several previous studies (Quack et al., 1999; Zhou et al., 1999; Yoshida et al., 2002; Napierala et al., 2005; Kim et al., 2006; Xuan et al., 2008; Lin et al., 2011). In these studies, most patients showed moderate to severe phenotypes such as hypoplastic or absent clavicles and delayed closure of cranial fontanels. R225 is located at the c-terminal region of the Runt domain, which has been shown to be associated with severe parietal bone dysplasia (Cunningham et al., 2006). The presence of the highly conserved DNA-binding domain, known as the Runt domain, indicates that the RUNX2 protein binds to a consensus DNA sequence. The Runt domain also mediates binding to CBF $\beta$, an unrelated partner protein that does not interact directly with DNA but enhances the DNA-binding affinity of the RUNX protein (Bartfeld et al., 2002). Substitution of R225 with glutamine is predicted to impair DNA binding to RUNX2. Quack et al. (1999) and Yoshida et al. (2002) have demonstrated that mutations at this codon interfere with nuclear localization and abolish DNA binding. Therefore, it is possible that the p.R225Q mutation affects the DNA-binding activity of RUNX2, causing the severe phenotypes in CCD. 
All affected individuals showed bilaterally absent clavicles, which was unusual because complete absence of the clavicles is rare and any absence of clavicles is usually limited to the acromial end (Shen et al., 2009). Dental abnormalities are another very common feature of CCD (Quack et al., 1999; Lo Muzio et al., 2007). In the affected individuals examined in this study, supernumerary teeth were located mainly in the anterior and premolar region, which is consistent with previous reports (Ishii et al., 1998). Besides the two abnormalities mentioned, the proband showed a median pseudo-cleft palate, a feature that has not been previously reported for this mutation. RUNX2 is the key transcription factor for osteoblastic differentiation, chondrocyte maturation, and skeletal morphogenesis (Ducy et al., 1997; Komori et al., 1997). RUNX2-cleft palate association has been reported in several previous studies. RUNX2 mutant mice embryos display failed fusions of the shelves of the secondary palate, which may lead to cleft palate in mice (Aberg et al., 2004). In a cohort study of 116 affected individuals and 62 controls, Cooper et al. (2001) observed a significantly increased prevalence of submucosal cleft palate. Moreover, Sull et al. (2008) suggested that the RUNX2 gene may be a risk factor for cleft palate in humans through a parent-of-origin effect consisting of excess maternal transmission. However, to date, no study has reported that the p.R225Q mutation in RUNX2 leads to cleft palate in humans.

In conclusion, this is the first report that provides structural evidence that the p.R225Q mutation might abolish DNA binding. This study has also shown that CCD-affected individuals with the same genotype have different CCD phenotypes, suggesting the other genetic and/ or environmental factors may affect the phenotypes of CCD.

\section{ACKNOWLEDGMENTS}

Research supported by grants from the National Natural Science Foundation of China (NSFC grants \#30973315 and \#81170964). We thank the patients for their cooperations. We also would like to give our special thanks to Prof. Tao Cai for his encouragement and support.

\section{REFERENCES}

Aberg T, Cavender A, Gaikwad JS, Bronckers AL, et al. (2004). Phenotypic changes in dentition of Runx2 homozygotenull mutant mice. J. Histochem. Cytochem. 52: 131-139.

Bartfeld D, Shimon L, Couture GC, Rabinovich D, et al. (2002). DNA recognition by the RUNX1 transcription factor is mediated by an allosteric transition in the RUNT domain and by DNA bending. Structure 10: 1395-1407.

Combet C, Jambon M, Deleage G and Geourjon C (2002). Geno3D: automatic comparative molecular modelling of protein. Bioinformatics 18: 213-214.

Cooper SC, Flaitz CM, Johnston DA, Lee B, et al. (2001). A natural history of cleidocranial dysplasia. Am. J. Med. Genet. 104: $1-6$.

Cunningham ML, Seto ML, Hing AV, Bull MJ, et al. (2006). Cleidocranial dysplasia with severe parietal bone dysplasia: C-terminal RUNX2 mutations. Birth Defects Res. A Clin. Mol. Teratol. 76: 78-85.

Ducy P, Zhang R, Geoffroy V, Ridall AL, et al. (1997). Osf2/Cbfa1: a transcriptional activator of osteoblast differentiation. Cell 89: 747-754.

Ishii K, Nielsen IL and Vargervik K (1998). Characteristics of jaw growth in cleidocranial dysplasia. Cleft. Palate Craniofac. J. 35: 161-166.

Kim HJ, Nam SH, Kim HJ, Park HS, et al. (2006). Four novel RUNX2 mutations including a splice donor site result in the cleidocranial dysplasia phenotype. J. Cell Physiol. 207: 114-122.

Komori T, Yagi H, Nomura S, Yamaguchi A, et al. (1997). Targeted disruption of Cbfa1 results in a complete lack of bone formation owing to maturational arrest of osteoblasts. Cell 89: 755-764.

Lee B, Thirunavukkarasu K, Zhou L, Pastore L, et al. (1997). Missense mutations abolishing DNA binding of the 
osteoblast-specific transcription factor OSF2/CBFA1 in cleidocranial dysplasia. Nat. Genet. 16: 307-310.

Lin WD, Lin SP, Wang CH, Tsai Y, et al. (2011). RUNX2 mutations in Taiwanese patients with cleidocranial dysplasia. Genet. Mol. Biol. 34: 201-204.

Lo Muzio L, Tete S, Mastrangelo F, Cazzolla AP, et al. (2007). A novel mutation of gene CBFA1/RUNX2 in cleidocranial dysplasia. Ann. Clin. Lab. Sci. 37: 115-120.

Mundlos S (1999). Cleidocranial dysplasia: clinical and molecular genetics. J. Med. Genet. 36: 177-182.

Mundlos S, Otto F, Mundlos C, Mulliken JB, et al. (1997). Mutations involving the transcription factor CBFA1 cause cleidocranial dysplasia. Cell 89: 773-779.

Napierala D, Garcia-Rojas X, Sam K, Wakui K, et al. (2005). Mutations and promoter SNPs in RUNX2, a transcriptional regulator of bone formation. Mol. Genet. Metab. 86: 257-268.

Otto F, Thornell AP, Crompton T, Denzel A, et al. (1997). Cbfa1, a candidate gene for cleidocranial dysplasia syndrome, is essential for osteoblast differentiation and bone development. Cell 89: 765-771.

Quack I, Vonderstrass B, Stock M, Aylsworth AS, et al. (1999). Mutation analysis of core binding factor A1 in patients with cleidocranial dysplasia. Am. J. Hum. Genet. 65: 1268-1278.

Shen Z, Zou CC, Yang RW and Zhao ZY (2009). Cleidocranial dysplasia: report of 3 cases and literature review. Clin. Pediatr. 48: 194-198.

Sull JW, Liang KY, Hetmanski JB, Fallin MD, et al. (2008). Differential parental transmission of markers in RUNX2 among cleft case-parent trios from four populations. Genet. Epidemiol. 32: 505-512.

Xuan D, Li S, Zhang X, Hu F, et al. (2008). Mutations in the RUNX2 gene in Chinese patients with cleidocranial dysplasia. Ann. Clin. Lab. Sci. 38: 15-24.

Yoshida T, Kanegane H, Osato M, Yanagida M, et al. (2002). Functional analysis of RUNX2 mutations in Japanese patients with cleidocranial dysplasia demonstrates novel genotype-phenotype correlations. Am. J. Hum. Genet. 71: 724-738.

Zhou G, Chen Y, Zhou L, Thirunavukkarasu K, et al. (1999). CBFA1 mutation analysis and functional correlation with phenotypic variability in cleidocranial dysplasia. Hum. Mol. Genet. 8: 2311-2316. 